\title{
Desorption of Hydrogen from the Steps on the Miscut Si(111) Surface Studied by SFG Spectroscopy
}

\author{
Goro Mizutani, ${ }^{\mathrm{a},}{ }^{\dagger}$ Zhipeng Yong, ${ }^{\mathrm{a}}$ Khuat Thi Thu Hien, ${ }^{\mathrm{a}}$ Harvey N. Rutt ${ }^{\mathrm{b}}$ \\ a School of Materials Science, Japan Advanced Institute of Science and Technology, 1-1 Asahidai, Nomi, Ishikawa 923-1292, Japan \\ $\mathrm{b}$ School of Electronics and Computer Science, University of Southampton, Southampton SO17 1BJ, U.K. \\ †Corresponding author:mizutani@jaist.ac.jp
}

Received: 13 December, 2019, Accepted: 26 April, 2020, Published: 7 May, 2020

We used sum frequency generation (SFG) spectroscopy to study the desorption of hydrogen on the stepped $\mathrm{Si}(111)$ surface with a miscut angle of $9.5^{\circ}$ toward the [11 2$]$ direction in ultra-high vacuum. The terrace peak was observed at $2085 \mathrm{~cm}^{-1}$ and the vertical step mode of dihydride was observed at $2098 \mathrm{~cm}^{-1}$ for the ssp-polarization (s-polarized SFG, s-polarized visible, and p-polarized infrared light) combination. The intensity reduction of these two peaks was monitored directly as a function of time at sample temperatures of $593 \mathrm{~K}$. The rate of reduction was faster for the step dihydride peak than for the terrace monohydride peak.

Keywords Optical sum frequency generation (SFG); Hydrogenated and stepped Si(111) surface; Dihydride; Desorption activation energy

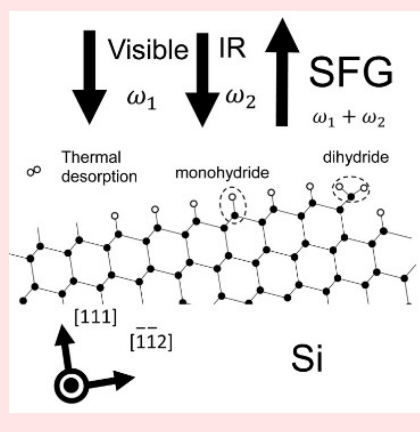

\section{INTRODUCTION}

Silicon is excellent as a semiconductor material and has been widely used. The hydrogen terminated silicon (H-Si) single crystal is an important model system of adsorbed semiconductor surfaces. In device and sensor fabrication processes, a $\mathrm{H}-\mathrm{Si}$ surface is first created before depositing ultra-thin functional films on it. Therefore, adsorption and desorption of hydrogen is very important in order to ensure the quality of ultra-thin films on $\mathrm{Si}$ [1].

There have been a number of studies of hydrogen desorption from $\mathrm{Si}(111)$ surfaces. It is generally known that the Si surface shows $\beta_{1}$ and $\beta_{2}$ peaks of $H_{2}$ desorption in TPD (temperature programmed desorption) spectra at around 783 and $673 \mathrm{~K}$, respectively [2]. The order of desorption of this system was determined by isothermal desorption at various temperatures around and below these peak temperatures. Second order desorption of hydrogen was reported by Schulze et al. [3] on the H-Si(111) $2 \times 1$ surface by TPD, and by Koeler et al. [4] and Wise et al. [5] on the H-Si(111) $7 \times 7$ surface by LITD (laser induced thermal desorption). They reported desorption energies from 2.5 to $2.73 \mathrm{eV}$. Desorptions with lower orders were reported by other researchers. Reider et al. [6] and Mao et al. [7] used SHG (optical second harmonic generation) and SFG (optical sum frequency generation), got the desorption order of $m=1.5$ at coverages lower than $0.05 \mathrm{ML}$ and obtained a desorption energy of around $2.4 \mathrm{eV}$. Morita et al. observed $\mathrm{H}-\mathrm{Si}(111) 1 \times 1$ by STM (scanning tunneling microscopy) and found that the desorption order changes from 2 to 1 as the coverage decreases [8]. They reported a desorption energy of $2.89 \mathrm{eV}$ for the $\sqrt{3} \times \sqrt{3} \mathrm{R} 30^{\circ}$ structure of the $\mathrm{Si}(111)$ surface. Sattar et al. used combined SFG and SHG methods and found that the desorption order switches from second to first at a coverage around 0.18 ML [9-11].

These reports are mostly giving a consistent picture, but some are partly inconsistent with each other, especially about the assignment of the TPD desorption peaks. The $\beta_{1}$ and $\beta_{2}$ peaks in TPD spectra are commonly assigned to $\mathrm{H}_{2}$ desorption from monohydrides and higher hydrides, respectively [12]. However, Tsetseris et al. reported a DFT study that the desorption energy of $3 \mathrm{H}$ complex from the $\mathrm{Si}(111)$ surface with excess hydrogen is $1.8 \mathrm{eV}$ [12], and it explains the behavior of the $\beta_{2}$ peak. According to his model the $\beta_{2}$ peak should show first order desorption as a function of the excess hydrogen coverage above $1 \mathrm{ML}$. He predicts surface vibrational S-H peaks at 2110 and $2154 \mathrm{~cm}^{-1}$. In contrast, Kim et al. adsorbed deuterium (D) on the $\operatorname{Si}(113)$ surface and observed desorption of $\mathrm{D}_{2}$ by the TPD method [13]. The $\beta_{2}$ peak around $673 \mathrm{~K}$ shows second order kinetics as it is judged from the analysis of the TPD curves, and the desorption energy is $2.16 \mathrm{eV}$. They suggested that this $\beta_{2}$ peak 
originates from dideuteride adatoms. During the $\mathrm{D}_{2}$ TPD experiment these dideuteride adatoms are converted to monodeuteride adatoms below $723-773 \mathrm{~K}$ and emit $\mathrm{D}_{2}$ molecules. Here, there is no consensus in the literature on the origins of $\beta_{2}$ peak in the TPD spectra and more research is necessary for better understanding of this $\mathrm{H}-\mathrm{Si}(111)$ system.

In this context, we tried to make progress in realizing a direct observation of monohydride and dihydride hydrogen on a $\mathrm{Si}$ surface by the SFG method while desorption of hydrogen is taking place. Optical SFG used in this study is one of the second-order nonlinear optical processes. It is a conversion process of visible light of frequency $\omega_{1}$ and infrared light of frequency $\omega_{2}$ into photons of sum frequency $\omega_{1}+\omega_{2}$. One feature of SFG spectroscopy is its symmetry selection rule. It selects only non-centrosymmetric parts of the sample within the dipole approximation with respect to the interaction of light with matter. Its sensitivity to surfaces and interfaces is much better than one monolayer [14], although this depends on the material system. Another feature is that it provides excellent vibrational spectroscopic data, as one can get resonant vibrational spectra of adsorbed atoms or molecules on surfaces when the infrared frequency is scanned. Since SFG gives a direct signal from step dihydride, we should be able to clarify the variation of its number density as a surface phenomenon progresses.

In this study, we aim to put a first step leading to a direct and sensitive method to dynamically monitor the hydrogen on the terraces and steps on the $\mathrm{Si}(111)$ stepped surface. In our past papers by SFG we studied hydrogen desorption kinetics of a hydrogenated $\mathrm{Si}(111)$ surface $[9,11]$ and a polarization dependence of the SFG spectra of a hydrogenated and stepped $\mathrm{Si}(111)$ surface. In this paper we have used $\mathrm{Si}(111)$ surface with a miscut angle of $9.5^{\circ}$ toward the [1112] direction. Here, we note that the step dihydride as illustrated in Figure 1(b) can contribute to the $\beta_{2}$ desorption peak. We monitored the signals from the terraces and steps on the Si(111) stepped surface directly and separately by measuring the SFG spectrum of the surface after adsorbing hydrogen on the surface.

\section{EXPERIMENT}

A stepped n-type phosphorus-doped $\mathrm{Si}(111)$ wafer with resistivity of $\sim 10 \Omega \mathrm{cm}$ was purchased from CrysTec $\mathrm{GmbH}$ with $9.5^{\circ}$ miscut angle toward the [1112] direction as schematically illustrated in Figure 1(b). The $20 \mathrm{~mm} \times 5 \mathrm{~mm} \times$ $0.1 \mathrm{~mm}$ samples for the measurement were cut from this wafer by a dicing saw (ACCRETECH A-WD-10B). According to literature [15], this miscut on the $\mathrm{Si}(111)$ surface is expected to induce more than $70 \%$ of the surface area to have triple steps. In Figure 1(b) a part of a triple step is seen at the right end of the figure.

In order to control the atomic structure of the Si surface, the sample temperature was controlled by direct current heating. The largest current was up to $6 \mathrm{~A}$ when the sample was heated to $1273 \mathrm{~K}$. First, the silicon plates with native oxide layers were heated at $873 \mathrm{~K}$ for $6 \mathrm{~h}$ to remove impurities. Next, the Si sample was flash heated at $1273 \mathrm{~K}$ for $60 \mathrm{~s}$ to form a reconstructed structure of $\mathrm{Si}(111) 7 \times 7$. Thereafter, the temperature of the Si sample was lowered to $873 \mathrm{~K}$, and pure molecular hydrogen gas was introduced into the chamber with the pressure of 3.5 Torr [7]. Hydrogen was supplied through a stainless steel tube dipped in a liquid nitrogen cooled trap. By cooling the $\mathrm{H}_{2}$ gas at liquid nitrogen temperature $77 \mathrm{~K}$, the impurity (mainly water molecules) in the gas was solidified so that high purity hydrogen was supplied into the chamber. There is a report that hydrogen adsorbs not only on the $\mathrm{Si}(111)$ face of the terrace but also on the step [13, 16-18]. After this adsorption treatment of about 15 min the sample was cooled down to room temperature. The SFG spectra were taken after the $\mathrm{H}-\mathrm{Si}(111)$ surface was heated at $593 \mathrm{~K}$ repeatedly for each $10 \mathrm{~s}$. The atomic structure of the Si surface was checked by LEED (OCI Vacuum Microengineering).

The optical setup of our SFG measurement was the same as that reported in our previous publication [9]. We used a $\mathrm{Nd}^{3+}$ :YAG laser (EKSPLA PL2143B) combined with a harmonic unit and OPG / OPA / DFG system (PG401/ DFG2-18P) as a light source with output light pulses of 30 ps width and $10 \mathrm{~Hz}$ repetition rate. The pulse energies of the IR and visible light were 100 and $10 \mu \mathrm{J}$, respectively. The sample was irradiated with visible light of $532 \mathrm{~nm}$ and infrared light of 4700 to $4900 \mathrm{~nm}$ at the same timing and at the same position. SFG light was collected and focused on the entrance slit of a monochromator (JASCO CT-25CD) equipped with a photomultiplier (Hamamatsu R-585) and collection optics. The strong background of the two incident light beams was removed by using a dichroic mirror and a band pass filter in the collection optics. The electric signals

(a) Macroscopic top view

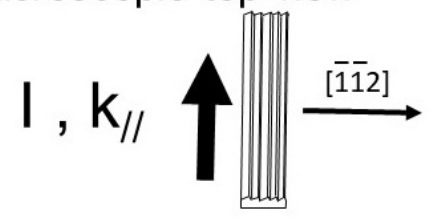

(b) Microscopic side view

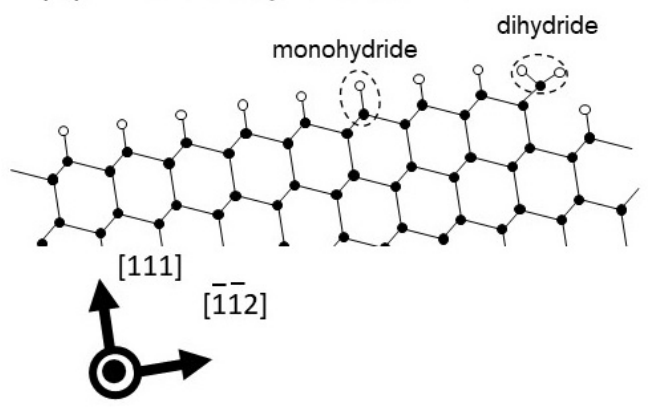

Figure 1: (a) Macroscopic and (b) microscopic structures of the hydrogenated stepped Si sample surface. $I$ represents the heating current direction for sample cleaning and $k \|$ represents the $k$-vector component parallel to the surface of the incident optical beams. 


\section{eJSSNT}
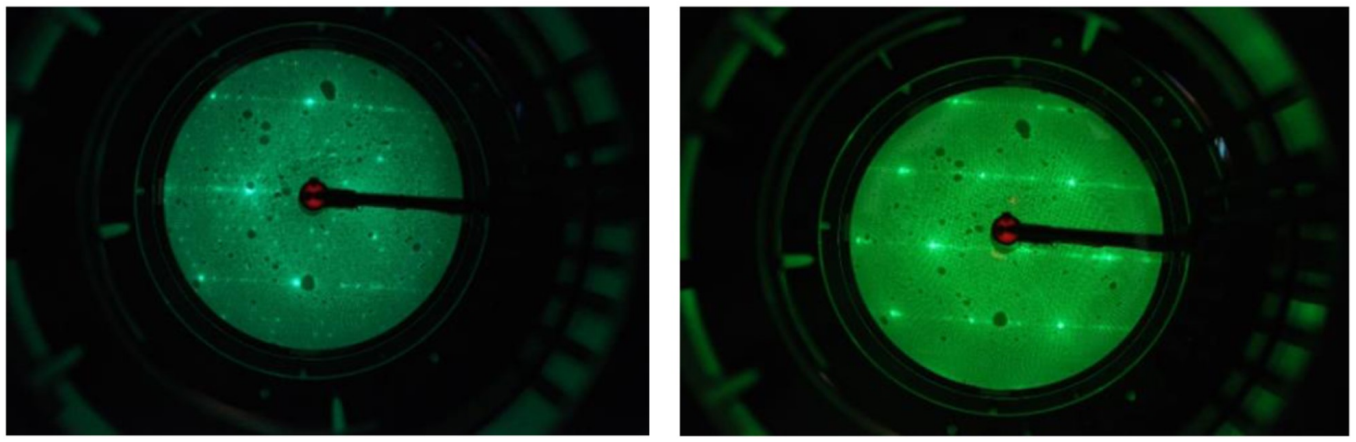

Figure 2: LEED patterns of hydrogenated and stepped $\mathrm{Si}(111)$ surfaces with a miscut angle of $9.5^{\circ}$ toward the [1̄1 2$]$ direction showing $(7 \times 7)$ (left) and $(1 \times 1)$ (right) surfaces before and after hydrogen adsorption.

from the photomultiplier recovered by a BOXCAR integrator and were stored in a computer. Signals from 300 pulses were accumulated at each wavenumber.

\section{RESULTS AND DISCUSSION}

Figure 2 shows the LEED patterns of the Si(111) miscut surface before and after the molecular hydrogen exposure. In Figure 2(a), we see diffraction spots of $7 \times 7$ reconstructed structure of $\mathrm{Si}(111)$ terraces created by flash heating at $1273 \mathrm{~K}$. The clear $7 \times 7$ structure indicates that the surface was clean before being exposed to molecular hydrogen. We also see streaks in the horizontal direction because of the periodic terrace and step structures. The center of the hexagonal $1 \times 1$ pattern is shifted from the center of the LEED screen. This is because of the miscut angle of the $\mathrm{Si}(111)$ substrate of $9.5^{\circ}$. In Figure 2(b), we see diffraction patterns of the $1 \times 1$ structure associated with streaks, but the $7 \times 7$ spots have disappeared due to the transition to $1 \times 1$ hydrogenated structure on the terraces. The intensity distribution in the streaks are consistent with the suggested triple step structure of our surfaces [19].

SFG intensity spectra for ssp-polarization (s-polarized SFG, s-polarized visible, and p-polarized infrared light) combination were obtained as a function of the IR wavenumber from 2060 to $2120 \mathrm{~cm}^{-1}$ in Figure 3. Two peaks are seen at $2085 \mathrm{~cm}^{-1}$ (A) and $2098 \mathrm{~cm}^{-1}(\mathrm{C} 1)$ in the spectra [16-18]. These peaks are assigned to monohydride $(\mathrm{SiH})$ and vertical step dihydride $\left(\mathrm{SiH}_{2}\right)$ modes, respectively, as they are illustrated in Figure 1(b).

In order to measure the speed of desorption of terrace and step hydrogen, the ssp-SFG spectra were taken repeatedly after each $10 \mathrm{~s}$ heating at $593 \mathrm{~K}$ until the total heating time reached $600 \mathrm{~s}$. Some of the SFG spectra obtained are shown in Figure 3. The black dots are experimental data and curves are the result of fitting. In Figure 3 , we see that the SFG intensities of the two peaks gradually reduce over time. These two peaks were separated by fitting the function [20],

$$
\left|\chi^{\mathrm{SFG}}\right|^{2}=\left|\chi^{\mathrm{NR}}+\frac{A_{1} e^{i \theta_{1}}}{x-\omega_{1}+i \gamma_{1}}+\frac{A_{2} e^{i \theta_{2}}}{x-\omega_{2}+i \gamma_{2}}\right|^{2},
$$

to the observed SFG intensity under the constraint of $\left|\theta_{1}-\theta_{2}\right|<0.2$ and the separated peak intensities of the two modes are plotted in Figure 4. Here we note that the asymmetry of the A peak at $t=0 \mathrm{~s}$ is reproduced well by the calculation and so it is because of the overlapping Lorentzian responses of the two oscillators. From both Figures 3 and 4, the step mode intensity is seen to approach zero faster than the terrace mode intensity. A similar result with a faster desorption rate was obtained for the sample temperature of $673 \mathrm{~K}$ (not shown).

Miyauchi et al. once reported a red-shift of the A peak of $\mathrm{Si}-\mathrm{H}$ vibration on a $\mathrm{H}-\mathrm{Si}(111)$ surface without miscut by 2 $\mathrm{cm}^{-1}$ during the hydrogen desorption [21]. Unfortunately, in Figure 3, we could not reduce the statistical errors to recognize this shift. Miyauchi et al. also reported an increase of the widths of the A peaks at lower coverage [21]. Unfortunately, we could not recognize a systematic change of the widths of the $A$ or $C_{1}$ peaks as a function of the desorption time.
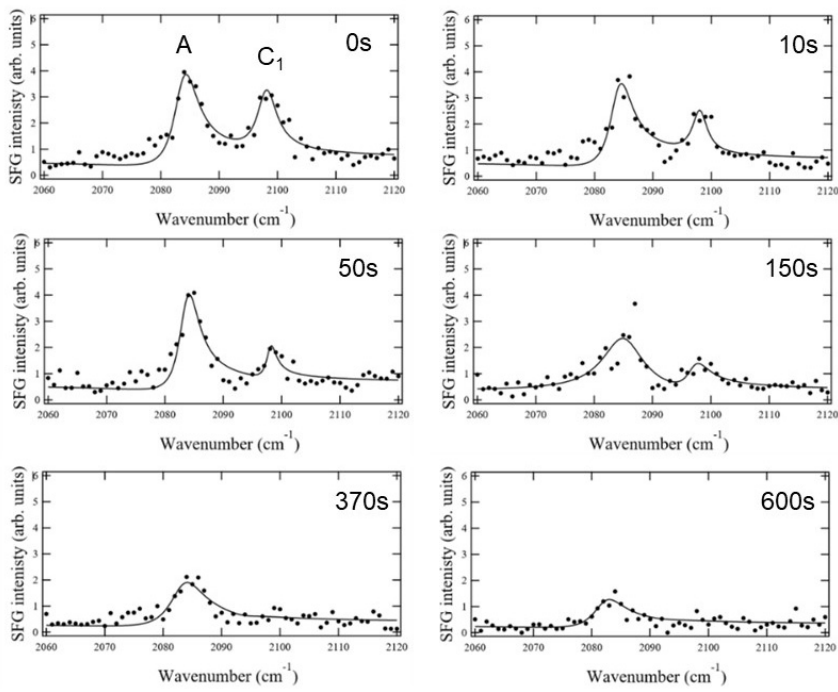

Figure 3: SFG spectra of the hydrogenated and stepped $\mathrm{Si}(111)$ surface with a miscut angle of $9.5^{\circ}$ toward the [112] direction in the ssp-polarization at various elapsed times for the heating temperature of $593 \mathrm{~K}$ (A and $\mathrm{C}_{1}$ peaks). 


\section{ALC '19 Conference}

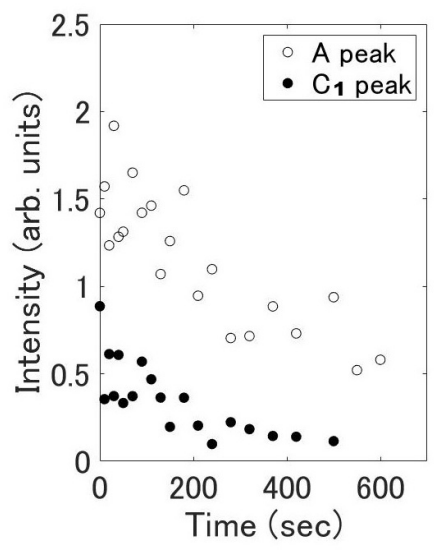

Figure 4: SFG intensity of monohydride $(\mathrm{A})$ and dihydride $\left(\mathrm{C}_{1}\right)$ as a function of time.

The results in this study should be compared with the work by Kim et al. mentioned in Section I [13]. In the study by Kim et al. crystalline $\mathrm{Si}(111)$ is miscut by $29.5^{\circ}$ in the [ 112] direction, while our sample is $\mathrm{Si}(111)$ with $9.5^{\circ}$ miscut in the same direction. So, the step edge lines run in the same crystallographic directions in the two samples. The A peak at $2085 \mathrm{~cm}^{-1}$ in Figure 3 is the vibration of $\mathrm{H}$ on the terrace $\mathrm{Si}$ adatom, and this hydrogen contributes to the $\beta_{1, \text { ad }}$ TPD peak. Therefore, the peak $\mathrm{A}$ is expected to show second-order desorption. Figure 5 shows the coverage of hydrogen on a logarithmic scale obtained from the A peak intensities in Figure 4 as a function of the heating time. Here, we used the result by Miyauchi et al. for the $\mathrm{H}-\mathrm{Si}(111)$ case without steps and converted the peak intensities to coverage under the effect of the dipole-dipole interaction [21-23]. Both the solid exponential curve corresponding to the first order desorption and the dashed curve of a function $1 /(a t+b)$ corresponding to the second order desorption can explain the coverage change, and we cannot deduce the desorption

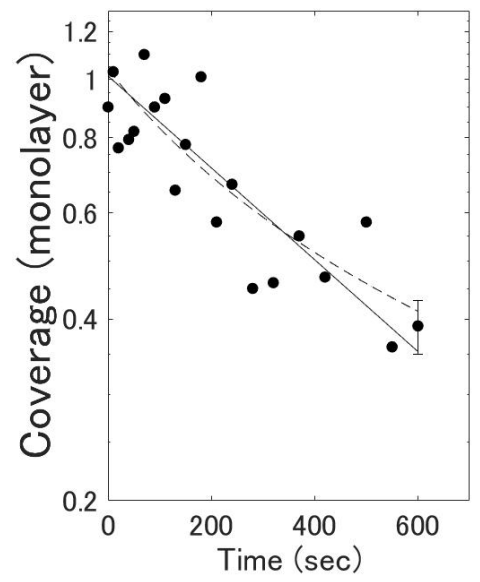

Figure 5: Coverage of terrace hydrogen calculated from the A mode intensity on a logarithmic scale vs. total heating time. The solid line indicates a best fit theoretical line for first order desorption and the dashed curve indicates a best-fit theoretical curve for second order desorption. order from our experiment. Based on the previous papers $[3-5,13]$, we guess that the A peak should follow the second order kinetics.

The $\mathrm{C}_{1}$ peak at $2098 \mathrm{~cm}^{-1}$ is the vibration of $\mathrm{H}$ on the step $\mathrm{Si}$. In the past literature researchers do not give any view as to whether this step hydrogen should contribute to $\beta_{1}$ or $\beta_{2}$ TPD peak. In Figure 4, the intensity of the $C_{1}$ peak at 2098 $\mathrm{cm}^{-1}$ approaches zero faster than that of peak A at $2085 \mathrm{~cm}^{-1}$. In Figure 6, we plotted the SFG intensity of the $C_{1}$ peak on a logarithmic scale as a function of heating time. We also drew fitted curves of first order desorption corresponding to an exponential decay (a solid line) and second order desorption corresponding to a decay as the function $1 /(a t+b)^{2}$ (a dashed line). Here the dipole-dipole interaction between step hydrogen atoms or between step and terrace hydrogen atoms is not considered for the following reason. The dipole-dipole interaction between the terrace $\mathrm{Si}-\mathrm{H}$ and step $\mathrm{Si}-\mathrm{H}_{2}$ bonds may not be negligible for higher coverage of hydrogen. The dipole-dipole interaction between step $\mathrm{Si}-\mathrm{H}_{2}$ bonds is probably weaker because their number density is smaller. On the other hand, in Figure 6, the two theoretical curves give a big difference for the time greater than $300 \mathrm{~s}$. For this time regime the dipole-dipole interaction is probably negligible because the number densities of both the terrace and step hydrogen are small. Hence, we did not consider the dipole-dipole interaction in analyzing Figure 6. Still, since there is a big error in the SFG intensity at very large times, the time variation of the $C_{1}$ peak intensity at $2098 \mathrm{~cm}^{-1}$ cannot be judged to be either first or second order.

The reason for the faster speed of approach to zero of the $\mathrm{C}_{1}$ SFG peak intensity might be two-fold. If the desorption of the $\mathrm{C}_{1}$ mode is of first order, this desorption order itself is the reason. The $\mathrm{C}_{1} \mathrm{SFG}$ intensity should reduce as an exponential function, while the A peak intensity should reduce as the function $1 /(a t+b)^{2}$. Then, the intensity of the $C_{1}$ peak should approach zero faster after a sufficiently long time.

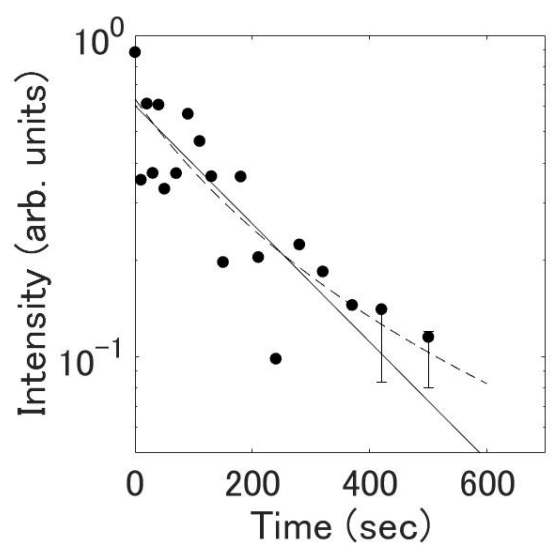

Figure 6: Step $\mathrm{C}_{1}$ mode SFG intensity on a logarithmic scale vs. total heating time. The solid line indicates a best fit theoretical line for first order desorption and the dashed curve indicates a best-fit theoretical curve for second order desorption. 
If the desorption of the $\mathrm{C}_{1}$ mode is in second order, the desorption energy from the step dihydride should be smaller than that of the terrace monohydride. Our desorption experiment at $673 \mathrm{~K}$ (not shown) gave a desorption rate constant around 45 times larger than that observed in Figure 6. This rate constant difference corresponds to a desorption activation energy of around $1.6 \mathrm{eV}$. So, we can roughly say that the desorption activation energy of the step hydrogen is smaller than that for the terrace hydrogen, since the latter is said to be around $2.5 \mathrm{eV}$.

According to Kim et al the $\beta_{2}$ TPD peak is due to excess hydrogen, but this is not necessarily consistent with the assignments by other researchers [2]. Some other papers argue that it is due to $\mathrm{CH}_{2}$ on defect sites on the Si surface [5]. SFG should have an advantage over TPD as a method for monitoring separately the desorption of hydrogen of different species. The assignment of the $\beta_{2}$ TPD peak can be determined more clearly if the desorption is measured in more detail by SFG than in the current work. Additionally, one can plan to carry out experiments at various temperatures and obtain the activation energies of the desorption more systematically. The study in this paper is intended as the first step for that future development.

\section{CONCLUSIONS}

In this study, we used sum frequency generation (SFG) spectroscopy to monitor the desorption of hydrogen from the stepped $\mathrm{Si}(111)$ surface with a miscut angle of $9.5^{\circ}$ toward [1 $1 \overline{1} 2]$ direction. The hydrogen was adsorbed by molecular dosing at a surface temperature of $873 \mathrm{~K}$. One terrace peak at $2085 \mathrm{~cm}^{-1}$ and another at $2098 \mathrm{~cm}^{-1}$ are assigned to the vertical step mode of dihydride, observed in the ssp-polarization. The SFG spectrum of the Si-H surface as a function of heating time at $593 \mathrm{~K}$ was obtained. The step peak intensity approached zero faster than the terrace peak intensity.

\section{Acknowledgments}

This work was supported by JSPS KAKENHI Grant Number JP15K05126.

\section{Note}

This paper was presented at the 12th International Symposium on Atomic Level Characterizations for New Materials and Devices '19 (ALC '19), in conjunction with the 22nd International Conference on Secondary Ion Mass Spectrometry (SIMS-22), Miyako Messe, Kyoto, Japan, 20-25 October, 2019.

\section{References}

[1] A. Vilan, O. Yaffe, A. Biller, A. Salomon, A. Kahn, and D. Cahen, Adv. Mater. 22, 140 (2010).
[2] P. Gupta, V. L. Colvin, and S. M. George, Phys. Rev. B 37, 8234 (1988).

[3] G. Schulze and M. Henzler, Surf. Sci. 124, 336 (1983).

[4] B. G. Koehler, C. H. Mak, D. A. Arthur, P. A. Coon, and S. M. George, J. Chem. Phys. 89, 1709 (1988).

[5] M. L. Wise, B. G. Koehler, P. Gupta, P. A. Coon, and S. M. George, Surf. Sci. 258, 166 (1991).

[6] G. A. Reider, U. Höfer, and T. F. Heinz, J. Chem. Phys. 94, 4080 (1991).

[7] M. Y. Mao, P. B. Miranda, D. S. Kim, and Y. R. Shen, Phys. Rev. B 64, 035415 (2001).

[8] Y. Morita, K. Miki, and H. Tokumoto, Surf. Sci. 325, 21 (1995). [9] Md. A. Sattar, K. T. T. Hien, Y. Miyauchi, G. Mizutani, and H. N. Rutt, Surf. Interface Anal. 48, 1235 (2016).

[10] K. T. T. Hien, M. A. Sattar, Y. Miyauchi, G. Mizutani, and H. N. Rutt, Surf. Sci. 663, 11 (2017).

[11] K. T. T. Hien, Y. Miyauchi, M. Kikuchi, and G. Mizutani, Surf. Interface Anal. 44, 662 (2012).

[12] L. Tsetseris and S. T. Pantelides, Phys. Rev. B 74, 113301 (2006).

[13] H. Kim, T. Spila, and J. E. Greene, Surf. Sci. 490, L602 (2001).

[14] P. Guyot-Sionnest, J. H. Hunt, and Y. R. Shen, Phys. Rev. Lett. 59, 1597 (1987).

[15] J. Wei, X.-S Wang, J. L. Goldberg, N. C. Bartelt, and E. D. Williams, Phys. Rev. Lett. 68, 3885 (1992).

[16] P. Jakob and Y. J. Chabal, J. Chem. Phys. 95, 2897 (1991).

[17] M. Morin, P. Jakob, N. J. Levinos, Y. J. Chabal, and A. L. Harris, J. Chem. Phys. 96, 6203 (1992).

[18] M. A. Hines, Y. J. Chabal, T. D. Harris, and A. L. Harris, Phys. Rev. Lett. 71, 2280 (1993).

[19] K. T. T. Hien, Z. Yong, G. Mizutani, and H. N. Rutt, to be published.

[20] Y. R. Shen and V. Ostroverkhov, Chem. Rev. 106, 1140 (2006).

[21] Y. Miyauchi, H. Chuat, and G. Mizutani, Surf. Sci. 614, 24 (2013).

[22] E. H. G. Backus and M. Bonn, Chem. Phys. Lett. 412, 152 (2005).

[23] M. Cho, C. Hess, and M. Bonn, Phys. Rev. B 65, 205423 (2002).

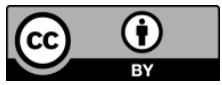

All articles published on e-J. Surf. Sci. Nanotechnol. are licensed under the Creative Commons Attribution 4.0 International (CC BY 4.0). You are free to copy and redistribute articles in any medium or format and also free to remix, transform, and build upon articles for any purpose (including a commercial use) as long as you give appropriate credit to the original source and provide a link to the Creative Commons (CC) license. If you modify the material, you must indicate changes in a proper way.

Published by The Japan Society of Vacuum and Surface Science 